\title{
Adaptive motion processing in bilateral vestibular failure
}

\author{
Roger Kalla, ${ }^{1,2,3}$ Neil Muggleton, ${ }^{2}$ Rainer Spiegel, ${ }^{1}$ Domenica Bueti, ${ }^{2}$ Jens Claassen, ${ }^{1}$ \\ Vincent Walsh, ${ }^{2}$ Adolfo Bronstein ${ }^{3,4}$
}

1 Department of Neurology and IFB ${ }^{L M U}$, University of Munich Hospital, Munich, Germany ${ }^{2}$ Institute of Cognitive Neuroscience, University College London, London, UK ${ }^{3}$ Department of Movement and Balance, Neuro-otology Unit, Charing Cross Hospital, Imperial College London, London, UK ${ }^{4}$ Department of Neuro-otology, National Hospital for Neurology \& Neurosurgery, London, UK

Correspondence to Adolfo Bronstein, Imperial College London, Charing Cross Hospital, London W6 8RF, UK: a.bronstein@imperial.ac.uk

Received 19 November 2010 Accepted 8 March 2011 Published Online First 7 May 2011

\section{ABSTRACT}

Background Patients with bilateral vestibular failure (BVF) suffer from oscillopsia during head movements. This is secondary to the loss of the vestibulo-ocular reflex which is responsible for stabilising retinal images during head movements of high frequency or velocity. Previous studies documented decreased visual motion sensitivity in such patients at low velocities. The authors now examine motion coherence tasks, which have two advantages: (1) the task is associated with the functions of the middle temporal area; and (2) it affords testing at low and high motion velocities, as relevant for patients with oscillopsia due to BVF.

Methods Nine BVF patients and nine healthy control subjects were examined with a random dot pattern with variable percentages of dots moving in the target direction. Participants were asked to indicate in which of two possible directions they perceived the coherent motion. Horizontal and vertical planes were tested at speeds from 0.156 to $40 \%$ s.

Results Motion coherence thresholds were lower at higher speeds in both groups $(p<0.0001)$. BVF patients had raised motion coherence thresholds $(p=0.002)$ across all velocities as compared with the control subject group.

Conclusion In a motion coherence paradigm, BVF patients show raised thresholds. This is the first demonstration of diminished visual motion processing at high velocities, supporting the view that the changes allow BVF patients to partly compensate for the oscillopsia. The findings are interpreted as an adaptive process likely to involve the middle temporal visual motion processing areas.

\section{INTRODUCTION}

Bilateral vestibular failure (BVF) is a chronic disorder of the peripheral labyrinths or the eighth cranial nerves. The patients present to the neurologist rather than to the otologist because patients usually have unsteadiness and visual symptoms, rather than hearing complaints. BVF can be caused by various aetiologies, such as progressive cerebellar ataxia, cranial or peripheral neuropathies, otological, neoplastic, autoimmune or associated neurological diseases; the more common causes are postmeningitis, ototoxic drugs (in particular gentamicin) and idiopathic BVF. ${ }^{12}$

A key symptom in BVF patients is oscillopsia, an illusionary movement of the visual world, during passive (eg, travelling in vehicles) and active head movements. Oscillopsia arises as a result of retinal image slip owing to an insufficient vestibulo-ocular reflex (VOR), the reflex responsible for stabilising retinal images during head movements. Several mechanisms exist in these patients that allow retinal image stability during slow head movements, such as pursuit-optokinetic and cervico-ocular reflexes. ${ }^{3-5}$ This is the reason why patients' oscillopsia is almost exclusively reported during activities involving head movements of high velocity or frequency content-for example, driving on a bumpy road, walking briskly or shaking the head.

During the acute stages of vestibular loss (eg, upon first mobilising after gentamicin treatment), oscillopsia is marked and distressing. However, with time, the severity of symptoms decreases, ${ }^{34}$ even though VOR recovery is exceptional, and oculomotor mechanisms hardly take over the highvelocity gaze-stabilising role of the VOR. ${ }^{4} 5$ This suggests that sensory or perceptual processes, in addition to oculomotor mechanisms, may contribute to the subjective symptomatic recovery of these patients.

In this regard, one study found that changes in subjective 'tolerance' to retinal image slippage was associated with lower oscillopsia handicap scores as a measure for defining the degree of disruption to the daily life and social activities of that individual ${ }^{6}$ and with a higher perceived degree of control over one's health. Although, at first, this finding is counterintuitive (ie, a higher retinal slip speed was associated with lower oscillopsia handicap scores), the suggestion was that adaptation to oscillopsia was partly dependent on the development of tolerance to the movement of images on the retina during selfmotion. Accordingly, the latter may be mediated by a reduction in visual-motion sensitivity. ${ }^{4} 6$

Evidence for a loss of sensitivity to visual motion was first described in patients with oculomotor disorders and was proposed to account for the rarity of reports of oscillopsia during head movements in these patients. ${ }^{7}$ It was later found that sensitivity for slowly moving small $\operatorname{targets}^{8}$ and drifting gratings ${ }^{9}$ was impaired in BVF patients, even when patients' heads were stationary, and the VOR was inactive. ${ }^{8}$ Consequently, the mechanism that helps to suppress oscillopsia during head movements cannot be entirely switched off when the head is held still, thus suggestive of a generalised adaptive suppression in motion sensitivity perception in these patients, presumably in cortical areas responsible for multimodal motion processing. ${ }^{8} 9$

A major limitation of previous studies measuring visual motion processing is that slow target velocities were used. Since pursuit-optokinetic mechanisms suffice to stabilise retinal images at low velocities, it could be argued that the velocities 
previously examined are neither relevant to the patients' oscillopsia nor relevant to the known physiology of the VOR. We therefore decided to examine visual motion perception with the motion coherence paradigm, ${ }^{9}$ where a random moving dot pattern is used. In addition to being able to examine a wide range of velocities, an added advantage of this task for the study of BVF patients is that motion coherence is thought to involve integration of information across the display, which is closely associated with activity of the middle temporal area of the brain. ${ }^{10-12}$ This is a visual area thought to be modified in patients with vestibular disorders, ${ }^{13-15}$ rather than areas more associated with earlier processing and local motion detection such as V1. ${ }^{11}$

\section{PATIENTS AND METHODS}

All participants gave their written informed consent before participating in the study.

\section{Patients}

We examined nine patients (three men, six women, aged $55-74$ years, mean $65 \pm 6.02$ years) with acquired chronic bilateral failure and nine healthy age-matched control subjects (aged $55-76$ years, mean $65.33 \pm 6.63$ years). Patients with BVF were determined by absent nystagmic responses on rotational (velocity step stimuli in darkness at $\pm 80^{\circ} / \mathrm{s}$ ) and bithermal caloric tests ( 30 and $44^{\circ} \mathrm{C}$, with and without visual fixation) as well as a bilateral pathological head impulse test, that is, clinically examnining the VOR by observing the patient's eyes during a small-amplitude but high-acceleration head turn. ${ }^{16}$ The aetiology of BVF was idiopathic $(n=6)$, bacterial meningitis $(n=2)$ and antibiotic gentamicin treatment $(n=1)$. The duration of oscillopsia and unsteadiness ranged from 8 to 26 years. Our control subjects had no history of labyrinthine, neurological and visual abnormalities. As tested with a Snellen chart, all patients and healthy control subjects had normal or corrected to normal vision.

\section{Procedure and experimental paradigms}

Subjects were required to judge the direction of motion of an array containing randomly positioned dots presented on a computer-controlled visual display unit with a refresh rate of $100 \mathrm{~Hz}$. A percentage of the dots moved coherently either up or down in one task (vertical task) or left or right in the other task (horizontal task). The remaining dots moved in random directions. The subject was required to indicate, by means of a key press, whether the coherent motion was up or down for the vertical task or left or right for the horizontal task. All subjects performed both tasks.

The tasks were programmed using the Cogent toolbox for Matlab (http://www.vislab.ucl.ac.uk/cogent.php), and the arrays were presented in a $30 \times 30^{\circ}$ area centred on the screen. Dots were white on a grey background $\left(35.8 \mathrm{~cd} . \mathrm{m}^{-2}\right)$ and faded to the background colour progressively in the last $6^{\circ}$ of the coherent motion axis. Arrays consisted of 1320 dots (for an illustration, see figure 1). The eye-screen distance was constant at $57 \mathrm{~cm}$, aided by a chin rest; subjects were asked to maintain fixation on a centrally presented '+' throughout each block.

To establish the subject's coherence threshold for a certain speed, a single-interval, two-option forced-choice procedure was used. Subjects were told beforehand whether the vertical or horizontal task was upcoming. For the vertical task, they were instructed that they would have to determine whether the motion direction was up or down, whereas for the horizontal task, they had to indicate whether it was left or right. Subjects practised usually four training blocks of 32 trials each until they

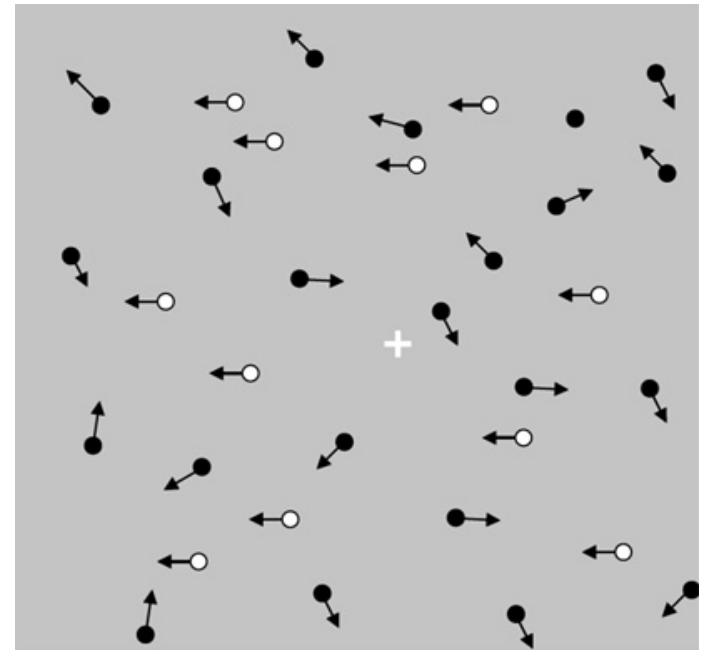

Figure 1 Array containing randomly positioned dots presented on a computer screen. A percentage of the dots moved coherently either up or down (vertical) or left or right (horizontal task). The remaining dots moved in random directions. The subject was required to indicate, by means of a key press, whether the coherent motion was up or down for the vertical task or left or right for the horizontal task. For illustrative purposes, coherent and randomly moving dots are indicated as white and black respectively.

were accustomed to the experiment. This included two training blocks for both directions (horizontal/vertical) with slow- and moderately fast-moving dots $(1.25$ and $10 \%$ s). The initial display contained dots in a one-to-one signal-to-noise ratio. If the observer's direction judgement was correct, the degree of coherent motion was decreased by $1 \mathrm{~dB}$. Likewise, an incorrect judgement led to an increase in the degree of coherent motion of $3 \mathrm{~dB}$. For each staircase, the observer completed 128 trials. To calculate a coherence threshold, the proportion of correct responses at each tested displacement was calculated. A probit analysis was used to find the coherence level at which the subject would be expected to perform at $75 \%$ correct. The dots moved at $0.15,0.3,0.6,1.25,2.5,5,10,20$ or $40^{\circ}$ s for both horizontal and vertical motion; hence 18 conditions were tested with 128 trials for each speed and direction combination. One speed and one axis of motion was used in each block of trials with the direction within each axis selected randomly across trials. The total testing time usually lasted $90 \mathrm{~min}$, including two breaks of $5 \mathrm{~min}$ each, in a room with normal lighting.

For statistical analysis, a repeated-measures ANOVA (Statistica 6.1, Statsoft) with post-hoc Scheffé tests was performed. The ANOVA design was composed of one between-subjects factor (group: normal/BVF patients) and two within-subjects factors (direction: horizontal/vertical; speed: 0.1562, 0.3125 , $0.625,1.25,2.5,5,10,20,40^{\circ} / \mathrm{s}$ ).

\section{RESULTS}

The task and an accompanying illustration appear in figure 1. The results for patients and control subjects are presented in figure 2 (for individual mean scores, see table 1), showing higher thresholds for patients than control subjects at all velocities and directions. The ANOVA on motion coherence thresholds revealed a significant main effect of group, $F(1,16)=12.432$, $\mathrm{p}=0.00281$, which was due to increased thresholds in patients. The main effect of speed, $F(8,128)=81.304, p<0.0001$, across both groups was due to a difference in thresholds as a function of target speed. Thresholds dropped steeply between 0.31 and 

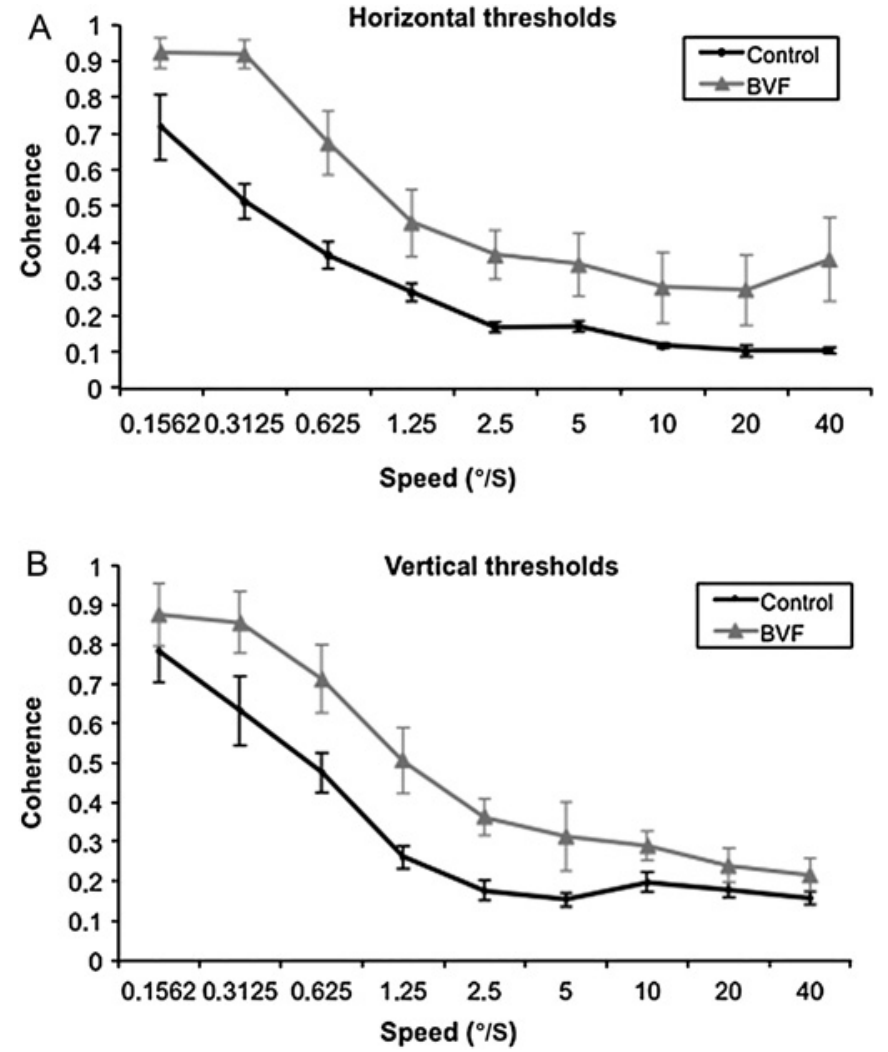

Figure 2 Analysis on horizontal-motion (A) and vertical-motion (B) coherence thresholds between bilateral vestibular failure (BVF) patients and healthy control subjects revealed a significant difference between patients and controls, and a significant effect of dot speed. Data are presented as mean and SE.

$2.5 \%$, and remained fairly stable thereafter. The ANOVA revealed no further significant main effects or interactions.

\section{DISCUSSION}

The present study tested motion coherence in patients with BVF and healthy control subjects, at a broad range of target speeds. This fulfilled two purposes. First, it allowed us to examine velocities of visual motion relevant for BVF patients (ie, that cannot be compensated for by cervico-ocular and/or pursuit optokinetic mechanisms. ${ }^{4}$ ) The result was that motion coherence thresholds were higher for patients than in the control group, that is, patients had more difficulty detecting coherent motion. Second, it allowed us to test if the ability to spot motion coherence was influenced by target velocity. Here, we found that higher target speeds have lower motion coherence thresholds in both groups, that is, they are easier to detect. The ANOVA conducted showed that there was no significant interaction between group and speed, indicating that patients and control subjects were equally affected by the various speeds. Similarly, there were no differences between vertical or horizontal visual motion. We will now discuss these two main findings.

First, the finding of higher motion coherence thresholds in patients with BVF suggests that compensation from bilateral loss of the VOR is a multifarious process, involving oculomotor, ${ }^{3} 4$ sensory ${ }^{59}$ and perceptual mechanisms. ${ }^{6}$ As mentioned in the Introduction, a number of studies are in line with this explanation. A study found that adaptation to retinal image slippage was associated with lower oscillopsia handicap scores, ${ }^{6}$ which was partly dependent on the development of tolerance to the movement of images on the retina during self-motion, suggestive of reduced visual motion sensitivity. ${ }^{4-9}$ Moreover, it was found that the mechanism responsible for suppressing oscillopsia during head movements is still active when the head is stationary. ${ }^{9}$ This result is in line with a generalised adaptive suppression in motion sensitivity perception in patients with BVF, which might take place in cortical areas responsible for multimodal motion processing. ${ }^{8} 9$ In order to extract the direction of motion in these motion tasks, the observer needs to combine information from many parts of the image, and it is therefore assumed to involve a global process rather than the local-motion detection of earlier stages in visual processing. ${ }^{10} 15$ Surprisingly, our results showed no direction effect, in contrast to data from Grünbauer and colleagues, ${ }^{8}$ who reported that horizontal object motion was more severely impaired than vertical motion perception. The difference between the two studies is likely to relate to the fact that Grünbauer and colleagues $^{8}$ focused on low speed thresholds. The low speed thresholds in these data (see figure 2A,B) also suggests there is a horizontal versus vertical effect found by Grünbauer and colleagues, as there is a greater difference between patients and healthy control subjects for horizontal rather than for vertical motion. As target speed increases (ie, with medium to high speed), this difference vanishes. Given that we tested a large range of thresholds, in contrast to earlier work where low thresholds only had been considered, it becomes clear why there is no overall direction effect.

As to which CNS areas may be involved in this adaptive process, functional brain-imaging studies with stimuli differing in their level of coherence show activity in the middle temporal area of the human brain but not in the preceding area of V1. ${ }^{10} 11$ In support of this notion, lesions to the middle temporal area of the macaque brain ${ }^{17}$ induce specific deficits for this task with relative sparing of other visual functions. ${ }^{10}{ }^{14}$ Further evidence stems from recent findings illustrating how vestibular signals contribute to cortical processes mediating self-motion perception. ${ }^{13}$ This study, which recorded the activity of neurons in the dorsal medial superior temporal area during a task in which monkeys combined visual and vestibular cues to discriminate heading, showed that responses recorded in dorsal medial superior temporal area were significantly correlated with the perceptual decisions of the monkeys and that the strongest correlations occurred in the most sensitive neurons. ${ }^{13}$ Consequently, the medial temporal area of the brain is active not only in the motion coherence paradigm but also in visuo-vestibular interaction, and thus is particularly relevant to our findings in patients with BVF. In contrast, a recent fMRI study on BVF patients ${ }^{18}$ showed no evidence of there being less activation in the middle temporal area, but this study did not use the motioncoherence paradigm. ${ }^{19} 20$ In another recent fMRI study with patients with unilateral vestibular failure, ${ }^{19}$ visual motion with optokinetic stimuli did show diminished activation of bilateral visual cortex areas, including the middle temporal area; this was interpreted as an adaptive mechanism suppressing oscillopsia. In agreement, we suggest that the raised coherent motion thresholds in BVF patients could be mediated by a downregulatory process in the middle temporal area leading to a subjective reduction in the levels of perceived oscillopsia during head movements. It should be taken into account that the time of testing since lesion onset varies strongly in our sample (8 to 26 years). This aspect could have led to possible reorganisation processes in some patients more than in others. Hence, we rather take on a cautious perspective by saying that downregulatory processes could have been at work, and that 
Table 1 Coherence thresholds at different speeds with horizontal $(A)$ and vertical $(B)$ motion for all bilateral vestibular failure (BVF) patients and healthy control subjects (Con)

\begin{tabular}{|c|c|c|c|c|c|c|c|c|c|}
\hline Speed $(\% / s)$ & 0.16 & 0.31 & 0.63 & 1.25 & 2.50 & 5.00 & 10.0 & 20.0 & 40.0 \\
\hline \multicolumn{10}{|l|}{ A } \\
\hline Con-1 & 0.93 & 0.24 & 0.24 & 0.16 & 0.13 & 0.14 & 0.11 & 0.10 & 0.10 \\
\hline Con-2 & 0.43 & 0.47 & 0.48 & 0.27 & 0.21 & 0.20 & 0.12 & 0.07 & 0.08 \\
\hline Con-3 & 1.00 & 0.70 & 0.35 & 0.31 & 0.16 & 0.22 & 0.17 & 0.10 & 0.11 \\
\hline Con-4 & 0.63 & 0.60 & 0.49 & 0.38 & 0.17 & 0.20 & 0.12 & 0.09 & 0.13 \\
\hline Con-5 & 0.47 & 0.51 & 0.41 & 0.30 & 0.15 & 0.22 & 0.11 & 0.15 & 0.07 \\
\hline Con-6 & 1.00 & 0.56 & 0.44 & 0.25 & 0.14 & 0.16 & 0.09 & 0.19 & 0.14 \\
\hline Con-7 & 0.81 & 0.37 & 0.17 & 0.13 & 0.13 & 0.10 & 0.09 & 0.07 & 0.08 \\
\hline Con-8 & 0.28 & 0.65 & 0.43 & 0.29 & 0.26 & 0.12 & 0.12 & 0.04 & 0.12 \\
\hline Con-9 & 0.90 & 0.51 & 0.27 & 0.28 & 0.14 & 0.16 & 0.11 & 0.10 & 0.08 \\
\hline BVF-1 & 0.98 & 0.75 & 0.44 & 0.32 & 0.27 & 0.17 & 0.13 & 0.10 & 0.18 \\
\hline BVF-2 & 1.00 & 0.99 & 0.56 & 0.27 & 0.22 & 0.18 & 0.12 & 0.18 & 0.28 \\
\hline BVF-3 & 1.00 & 0.87 & 0.51 & 0.31 & 0.25 & 0.21 & 0.10 & 0.12 & 0.10 \\
\hline BVF-4 & 1.00 & 1.00 & 1.00 & 0.76 & 0.66 & 1.00 & 1.00 & 1.00 & 1.00 \\
\hline BVF-5 & 0.67 & 0.71 & 0.29 & 0.17 & 0.15 & 0.26 & 0.09 & 0.13 & 0.13 \\
\hline BVF-6 & 1.00 & 1.00 & 1.00 & 0.88 & 0.68 & 0.26 & 0.10 & 0.40 & 0.88 \\
\hline BVF-7 & 1.00 & 1.00 & 0.89 & 0.79 & 0.53 & 0.46 & 0.43 & 0.25 & 0.33 \\
\hline BVF-8 & 0.78 & 1.00 & 0.86 & 0.31 & 0.30 & 0.29 & 0.29 & 0.12 & 0.10 \\
\hline BVF- 9 & 0.86 & 0.93 & 0.51 & 0.27 & 0.23 & 0.23 & 0.24 & 0.13 & 0.16 \\
\hline \multicolumn{10}{|l|}{ B } \\
\hline Con-1 & 1.00 & 0.75 & 0.44 & 0.16 & 0.14 & 0.15 & 0.28 & 0.09 & 0.15 \\
\hline Con-2 & 0.70 & 0.76 & 0.61 & 0.24 & 0.29 & 0.09 & 0.13 & 0.19 & 0.08 \\
\hline Con-3 & 1.00 & 1.00 & 0.48 & 0.30 & 0.28 & 0.24 & 0.17 & 0.21 & 0.14 \\
\hline Con-4 & 0.89 & 0.93 & 0.78 & 0.42 & 0.19 & 0.13 & 0.18 & 0.17 & 0.23 \\
\hline Con-5 & 1.00 & 0.71 & 0.41 & 0.34 & 0.22 & 0.21 & 0.25 & 0.20 & 0.15 \\
\hline Con-6 & 0.64 & 0.48 & 0.37 & 0.16 & 0.17 & 0.16 & 0.09 & 0.10 & 0.18 \\
\hline Con-7 & 0.39 & 0.19 & 0.41 & 0.31 & 0.13 & 0.11 & 0.20 & 0.16 & 0.23 \\
\hline Con-8 & 0.47 & 0.39 & 0.53 & 0.25 & 0.07 & 0.18 & 0.32 & 0.26 & 0.14 \\
\hline Con-9 & 0.94 & 0.48 & 0.24 & 0.17 & 0.10 & 0.09 & 0.15 & 0.22 & 0.11 \\
\hline BVF-1 & 0.96 & 0.60 & 0.39 & 0.24 & 0.28 & 0.27 & 0.24 & 0.18 & 0.17 \\
\hline BVF-2 & 1.00 & 0.85 & 0.79 & 0.35 & 0.45 & 0.18 & 0.21 & 0.14 & 0.21 \\
\hline BVF-3 & 1.00 & 0.99 & 0.58 & 0.48 & 0.37 & 0.98 & 0.21 & 0.13 & 0.10 \\
\hline BVF-4 & 1.00 & 0.91 & 1.00 & 0.46 & 0.40 & 0.25 & 0.30 & 0.46 & 0.30 \\
\hline BVF-5 & 0.54 & 0.34 & 0.24 & 0.17 & 0.11 & 0.13 & 0.17 & 0.05 & 0.11 \\
\hline BVF-6 & 1.00 & 1.00 & 0.79 & 0.91 & 0.44 & 0.15 & 0.51 & 0.30 & 0.48 \\
\hline BVF-7 & 1.00 & 1.00 & 0.82 & 0.59 & 0.59 & 0.38 & 0.43 & 0.33 & 0.29 \\
\hline BVF-8 & 0.39 & 1.00 & 1.00 & 0.50 & 0.23 & 0.17 & 0.22 & 0.21 & 0.17 \\
\hline BVF- 9 & 1.00 & 1.00 & 0.79 & 0.85 & 0.39 & 0.31 & 0.32 & 0.35 & 0.11 \\
\hline
\end{tabular}

this hypothesis would need to be tested in greater detail in the future.

Another interpretation that should not be neglected is that a loss of vestibular input can just as well directly lead to impaired motion perception, so instead of motion detection being an adaptive response, it could just as well be a consequence of the lesion. However, this interpretation would contradict the findings by Grunfeld et al in patients with BVF showing that patients who are better compensated display more tolerance to retinal slippage. ${ }^{6}$ This suggests that the decrease in visual motion sensitivity is indeed a secondary, compensatory process. An additional mechanism could be related to adaptation effects, which occur in normal subjects following presentation of moving stimuli. The contribution of aftereffects to reduced sensitivity in patient populations may also warrant further investigation.

The second finding of this paper was that motion coherence is detected more easily when motion is faster, both in healthy control subjects and in patients with BVF. This finding in control subjects is consistent with both the greater change in information inherent to faster-moving stimuli and previous reports of degraded motion discrimination for lower-speed stimuli presented for the same duration. ${ }^{21}$ While BVF patients were impaired relative to control subjects, they maintained a similar pattern of better performance for higher-speed coherent motion.

To conclude, this study showed that BVF patients have higher motion coherence thresholds than control subjects, which may be the result of an adaptive process to reduce unpleasant symptoms of oscillopsia owing to retinal image motion during head movements in patients with loss of the VOR. Stimulus speed influenced motion coherence thresholds, that is, motion coherence was spotted more easily when motion was faster in both subject groups.

Acknowledgements This experiment was carried out using Cogent Graphics developed by J Romaya at the LON at the Wellcome Department of Imaging Neuroscience, London, UK. We thank the patients and healthy control subjects who kindly agreed to take part in this study.

Funding This work was supported by the German Ministry of Education and Research (BMBF), grant No 01 E00901 to the IFBLMU 'Integriertes Forschungs- und Behandlungszentrum für Schwindel, Gleichgewichts- und Augenbewegungsstörungen' and a post-doc grant to the first author by Deutsche Forschungsgemeinschaft (DFG, 
Germany) in collaboration with the Medical Research Council (UK), Grant No KA2284/2-1.

\section{Competing interests None.}

Patient consent Obtained.

Ethics approval Ethics approval was provided by the University College London, Department of Cognitive Neuroscience Ethics Approval Board (ethics approval no EA1144/001)

Provenance and peer review Not commissioned; externally peer reviewed.

\section{REFERENCES}

1. Brandt T. Bilateral vestibulopathy revisited. Eur J Med Res 1996:24:361-8.

2. Rinne T, Bronstein AM, Rudge P, et al. Bilateral loss of vestibular function: clinical findings in 53 patients. J Neurol 1998;245:314-21.

3. Gresty MA, Hess K, Leech J. Disorders of the vestibulo-ocular reflex producing oscillopsia and mechanisms compensating for loss of labyrinthine function. Brain 1977:100:693-716.

4. Bronstein AM, Hood JD. Oscillopsia of peripheral vestibular origin. Central and cervical compensatory mechanisms. Acta Otolaryngol 1987;104:307-14.

5. Bronstein AM, Morland AB, Ruddock KH, et al. Recovery from bilateral vestibular failure: implications for visual and cervico-ocular function. Acta Otolaryngol Suppl 1995:520:405-7.

6. Grunfeld EA, Morland AB, Bronstein AM, et al. Adaptation to oscillopsia: a psychophysical and questionnaire investigation. Brain 2000;123:277-90.

7. Dieterich M, Brandt TH. Impaired motion perception in congenital nystagmus and acquired ocular motor palsy. Clin Vision Sci 1987;1:337-45.

8. Grünbauer WM, Dieterich M, Brandt T. Bilateral vestibular failure impairs visual motion perception even with the head still. Neuroreport 1998;9:1807-10.
9. Shallo-Hoffmann J, Bronstein AM. Visual motion detection in patients with absent vestibular function. Vision Res 2003:43:1589-94.

10. Snowden RJ, Kavanagh E. Motion perception in the ageing visual system. Minimum motion, motion coherence, and speed discrimination thresholds. Perception 2006; $35: 9-24$

11. Braddick OJ, O'Brien JMD, Wattam-Bell J, et al. Brain areas sensitive to coherent visual motion. Perception 2001;30:61-72.

12. Händel B, Lutzenberger $W$, Thier $P$, et al. Opposite dependencies on visual motion coherence in human area MT+ and early visual cortex. Cereb Cortex 2007:17:1542-9

13. Gu Y, DeAngelis GC, Angelaki DE. A functional link between area MSTd and heading perception based on vestibular signals. Nat Neurosci 2007:10:1038-47.

14. Newsome WT, Paré EB. A selective impairment of motion perception following lesions of the middle temporal visual area (MT). J Neurosci 1988:8:2201-11.

15. Morrone MC, Burr DC, Viana LM. Two stages of visual processing for radial and circular motion. Nature 1995; 376:507-9.

16. Halmagyi GM, Curthoys IS. A clinical sign of canal paresis. Arch Neurol 1988:45:737-9.

17. Snowden RJ. Motion processing in the primate cerebral cortex. In: Smith AT, Snowden RJ, eds. Visual Detection of Motion. London: Academic Press, 1994:51-83

18. Dieterich M, Bauermann T, Best C, et al. Evidence for cortical visual substitution of chronic bilateral vestibular failure (an fMRI study). Brain 2007;130:2108-16.

19. Deutschländer $\mathbf{A}$, Hüfner $\mathrm{K}$, Kalla $\mathrm{R}$, et al. Unilateral vestibular failure suppresses cortical visual motion processing. Brain 2008;131:1025-34.

20. Dieterich M, Brandt T. Functional brain imaging of peripheral and central vestibula disorders. Brain 2008:131:2538-52.

21. Lappin JS, Tadin D, Nyquist JB, et al. Spatial and temporal limits of motion perception across variations in speed, eccentricity, and low vision. J Vis 2009:9:1-14. 


\section{Adaptive motion processing in bilateral vestibular failure}

Roger Kalla, Neil Muggleton, Rainer Spiegel, Domenica Bueti, Jens

Claassen, Vincent Walsh and Adolfo Bronstein

J Neurol Neurosurg Psychiatry 2011 82: $1212-1216$ originally published online May 7, 2011

doi: 10.1136/jnnp.2010.235960

Updated information and services can be found at:

http://jnnp.bmj.com/content/82/11/1212

These include:

References This article cites 20 articles, 8 of which you can access for free at: http://jnnp.bmj.com/content/82/11/1212\#BIBL

Email alerting Receive free email alerts when new articles cite this article. Sign up in the service box at the top right corner of the online article.

Topic Articles on similar topics can be found in the following collections Collections

Ophthalmology (755)

\section{Notes}

To request permissions go to:

http://group.bmj.com/group/rights-licensing/permissions

To order reprints go to:

http://journals.bmj.com/cgi/reprintform

To subscribe to BMJ go to:

http://group.bmj.com/subscribe/ 